\title{
Chaos Analysis on the Acceleration Control Signals of the Piezoelectric Actuators in the Stewart Platform
}

\author{
Jiaqiang E, ${ }^{1,2}$ Cheng Qian, ${ }^{1,2}$ Teng Liu,, and Guanling Liu $^{1,2}$ \\ ${ }^{1}$ College of Mechanical and Vehicle Engineering, Hunan University, Changsha 410082, China \\ ${ }^{2}$ Institute of New Energy and Energy-Saving \& Emission-Reduction Technology, Hunan University, Changsha 410082, China
}

Correspondence should be addressed to Jiaqiang E; ejiaqiang@163.com

Received 14 May 2015; Revised 9 September 2015; Accepted 10 September 2015

Academic Editor: Vadim V. Silberschmidt

Copyright (C) 2016 Jiaqiang E et al. This is an open access article distributed under the Creative Commons Attribution License, which permits unrestricted use, distribution, and reproduction in any medium, provided the original work is properly cited.

In order to solve the nonlinear mechanism in the process of actual control application of six-degree-of-freedom parallel Stewart platform, the chaos theory was applied in the paper to analyze the acceleration control signal. The research included correlation dimension calculation by use of the G-P method, the maximum Lyapunov coefficient of the acceleration control signal, and Kolmogorov entropies of the acceleration control signal. The results show that the acceleration signals are of chaos characteristics, and there are lots of influencing factors to the acceleration variables.

\section{Introduction}

There are a lot of uncertainty factors and nonlinear mechanisms in the process of actual control application of sixdegree-of-freedom parallel Stewart platform [1-3]. It is very obvious that vibration characteristics of the new type of passive super static vibratory platform [4] are of prodigious complexity. Chang-Jian and Chang [5] perform a systematic analysis of the dynamic behavior of a single degreeof-freedom spur gear system with and without nonlinear suspension (resp.), in which the onset of chaotic motion is identified from the phase diagrams, power spectra, Poincaré maps, Lyapunov exponents, and fractal dimension of the gear system. The numerical results reveal that the system exhibits a diverse range of periodic, subharmonic, and chaotic behaviors. Li et al. [6] give a mathematically analytical proof on the existence of chaos in a generalized Duffingtype oscillator with fractional-order deflection and provide a rigorous theoretical foundation to support studies and applications of this important class of dynamical systems. Hu et al. [7] use a chaotic analysis method to analyze pressure signal of a slurry column in order to indicate the flow pattern. Gao et al. [8] identify time-delay fractional-order chaos systems via the differential evolution algorithms, and the experiments' results show that the proposed inversion mechanism for time-delay fractional-order chaotic systems is a successful method for the advantages of high precision and robustness. Farshidianfar and Saghafi [9] use Melnikov analysis to develop a practical model of gear system to control and eliminate the chaotic behavior. Gaume et al. [10] study the rainfall time series, it resulting from stochastic or low-dimensional deterministic chaotic processes; the authors used nonlinear analysis tools dedicated to the identification of chaotic behavior. Miandoab et al. [11, 12] study nonlinear dynamics and chaos in MEMS/NEMS resonators and indicate that the necessary condition for the creation of chaos in the resonator is the intersection of the system steady state response with the homoclinic orbit. Khatibi et al. [13] investigate the existence of chaotic behavior in the river stage and observe discharge time series at the Sogutluhan hydrometric station, Turkey. The results indicate the existence of low-dimensional chaos in the two time series. In order to complete identification of chaos of nonlinear nonholonomic systems, Chang and Ge [14] study the scope of the chaos; the most reliable Lyapunov exponents, phase portraits, Poincaré maps, and bifurcation diagrams are also proved. Yuan et al. [15] apply the 0-1 test for chaos to detect chaos exhibited by fractional-order delayed systems. Luo and Wang [16] investigate dynamic behaviors of a fractionalorder chaotic system in complex space. Mahmoud et al. [17] investigate the phenomenon of chaos synchronization of two different chaotic complex systems of the Chen and Lü type via 


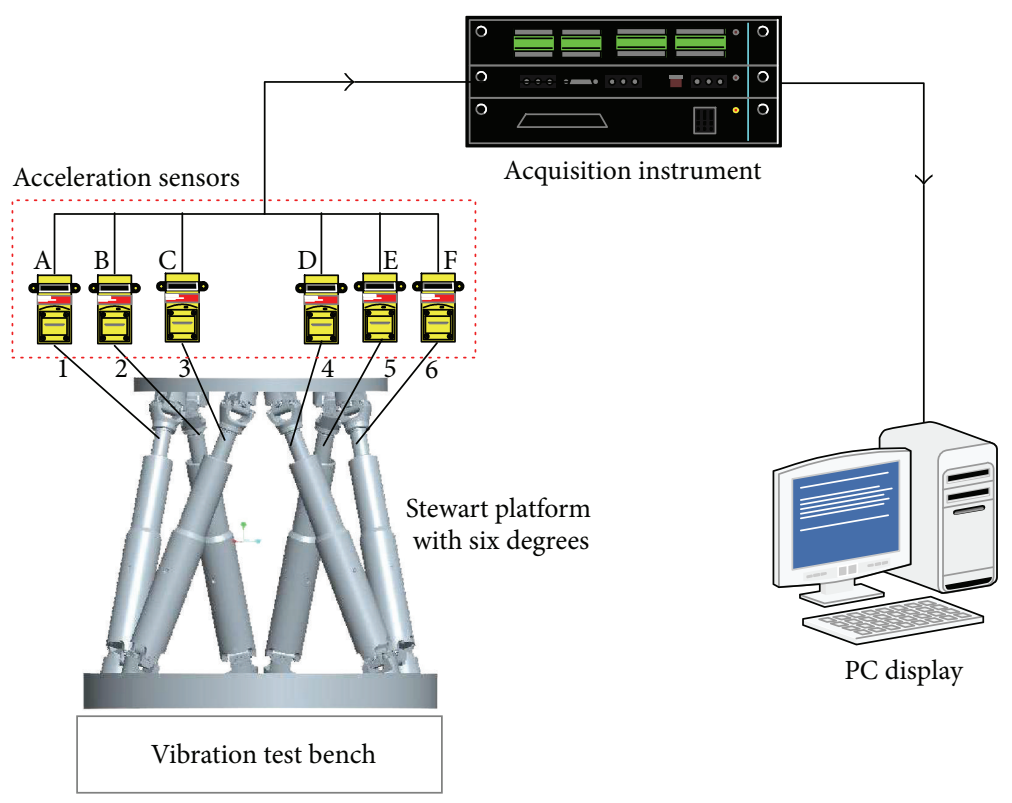

FIGURE 1: Test system of Stewart platform with six degrees.

the methods of active control and global synchronization. $\mathrm{Wu}$ and Baleanu [18] propose a discrete fractional logistic map from bifurcation diagrams; (in addition,) the chaotic behaviors are numerically illustrated. Chaos appears in nonlinear system mainly, and the classification of the nonlinear system is mainly composed of attractor for calibration, which is the core of the chaotic motions of the nonlinear dissipative system [19]. Li et al. [20, 21] study analytical control of chaos based on theoretical analysis and experimental verification and the research results which are of exciting significance are used to study on algebraic criteria for second-order global consensus in multiagent networks [22, 23].

Attractor refers to the motion of nonlinear dynamic system eventually formed in the phase space of the invariant manifold or point set; thus it approximately can be divided into three categories [24-26]: (1) A fixed point that is tracked through all phase spaces is named as the first kind of attractor that can describe calibration final state of the nonlinear time invariant system. (2) The phase space of the closed curve and curved surface is named as the second kind of attractor that can describe the state of the time variant system. (3) Besides, the other kinds of attractors are collectively regarded as strange attractors or chaotic attractors.

So for the vibration control of the piezoelectric actuator, the acceleration test data of the piezoelectric actuator must be analyzed. In order to take a precise control of the piezoelectric actuator, the nonlinear characteristic of the actuator must be known, so the control method can be designed based on the analyzed result. In the paper, the chaos algorithm was applied to distinguish whether the system was a chaos system or not. Chaos analysis for nonlinear system is mainly the study of the strange attractor, and its nature can be described as follows: the Lyapunov coefficient which represents the characteristic index of dynamic system, Kolmogorov entropy which represents the chaos level of the dynamic system, and the correlation dimension which stands for the complexity of the dynamic system. In order to determine whether a dynamic system is a chaotic system or not, the chaotic attractor must be provided with the following two basic characteristics: (1) The attractor of the system in the phase space has a structure of self-similar fractal dimension. (2) The system is sensitive to initial condition. If the conditions above can be available to the attractor, the system is of chaotic characteristic.

\section{Chaos Fractal Research on Acceleration Time Series Based on the Piezoelectric Actuator}

In order to establish a vibration control model of sixdegree-of-freedom parallel Stewart platform, the detection acceleration signals from the piezoelectric actuator load side are regarded as control reference, and the acceleration responses of piezoelectric actuator 1, piezoelectric actuator 2, piezoelectric actuator 3, piezoelectric actuator 4, piezoelectric actuator 5, and piezoelectric actuator 6 are recorded by using of acceleration sensor A, acceleration sensor $B$, acceleration sensor $\mathrm{C}$, acceleration sensor $\mathrm{D}$, acceleration sensor $\mathrm{E}$, and acceleration sensor $\mathrm{F}$ and data acquisition equipment based on the influences of the mechanical disturbances coming from the bottom of parallel Stewart platform as shown in Figure 1. The type of acceleration sensor is YD121-1000 ICP. The data acquisition equipment is LMS SCADAS Mobile. 
The acceleration values are shown in Figure 2 and acceleration time series are reconstructed to obtain the novel phase space of the attractors; then it can be identified whether acceleration time series are of chaotic characteristic or not.

2.1. Phase Space Reconstruction. The phase space reconstruction theory is put forward by Takens [27] and it can be used to analyze the chaotic characteristic of time series as a kind of low order nonlinear dynamic systems. Assuming that $x_{1}, x_{2}, \ldots, x_{j}, \ldots, x_{n}$ are acceleration time series of actuator as is shown in Figure 2 and $\tau$ is selected as delay time, $m$ for embedding dimension, then the reconstructed phase space was expressed as

$$
\begin{array}{r}
\mathbf{X}(t) \\
=\{x(t), x(t+\tau), x(t+2 \tau), \ldots, x[t+(m-1) \tau]\}^{T}, \\
t=1,2, \ldots, N,
\end{array}
$$

where $N$ represents phase points, $N=n-(m-1) \tau ; \mathbf{X}(t)$ represents dimensional phase space vector of $m$.

Then the phase space sequence composed by (1) is expressed as

$$
\begin{aligned}
& (\mathbf{X}(1) \quad \mathbf{X}(2) \cdots \mathbf{X}(j) \cdots \quad \mathbf{X}(N-(m-1) \tau))^{-1} \\
& \quad=\left[\begin{array}{ccccc}
x(1) & x(1+\tau) & x(1+2 \tau) & \cdots & x(1+(m-1) \tau) \\
x(2) & x(2+\tau) & x(2+2 \tau) & \cdots & x(2+(m-1) \tau) \\
\vdots & \vdots & \vdots & & \vdots \\
x(j) & x(j+\tau) & x(j+2 \tau) & \cdots & x(j+(m-1) \tau) \\
\vdots & \vdots & \vdots & & \vdots \\
x(N-(m-1) \tau) & x(N-(m-2) \tau) & x(N-(m-3) \tau) & \cdots & x\left(t_{n}\right)
\end{array}\right] .
\end{aligned}
$$

There are interactions in evolution among the components in (2); therefore, the information of the system is implied in the process of the development of each component; thus a certain change rule can be found and extracted from the system. As long as delay time $t$ and the embedding dimension $m$ are known, phase space can be reconstructed from (2).

It is well known that autocorrelation method, mean displacement, multiple correlation method, and mutual information are often used to calculate the delay time $\tau$ as the main methods. In the paper, the autocorrelation method is adopted and its computational formula is expressed as

$$
f(\tau)=\frac{\sum_{t=1}^{n-k}\left(x_{t}-\bar{x}\right)\left(x_{t+k}-\bar{x}\right)}{\sum_{t=1}^{n-k}\left(x_{t}-\bar{x}\right)^{2}},
$$

where $f(\tau)$ represents the $k$ order autocorrelation coefficient; $\bar{x}$ is the average value of $\left\{x_{t}\right\}$.

When the correlation coefficient is close to zero, the corresponding $k$ is the desired delay time $\tau$.

In the process of chaos study, how to determine whether the attractor is scale-free interval or fractal is a firstly important thing. Thus the concept of correlation dimension is brought by Grassberger and Procaccia, or G-P method for short. The calculation process is expressed as follows.

The integral function $C_{m}(\varepsilon)$ can be defined as [27]

$$
C_{m}(\varepsilon)=\frac{1}{N_{m}^{2}} \sum_{i=1}^{N_{m}} \sum_{j=1}^{N_{m}} H\left(\varepsilon-\left\|X_{i}-X_{j}\right\|\right),
$$

where $\varepsilon$ represents the selected distance; $\left\|X_{i}-X_{j}\right\|$ represents the Euclidean distance between $X_{i}$ and $X_{j} ; H$ represents the function of Heaviside.

Then $\varepsilon$ is selected; $C_{m}(\varepsilon)$ represents the probability of Euclidean distance between $X_{i}$ and $X_{j}$ less than $\varepsilon$; with the increase of $\varepsilon, C_{m}(\varepsilon)$ will increase as rate; the corresponding relation is expressed as

$$
C_{m}(\varepsilon) \propto \varepsilon^{D_{2}} .
$$

After both ends of (5) are taken by logarithm simultaneously, (6) can be obtained [27]:

$$
\ln C_{m}(\varepsilon) \propto D_{2} \ln \varepsilon .
$$

From (6), in scale-free interval, the relationship between $\ln C_{m}(\varepsilon)$ and $\ln (\varepsilon)$ is linear; the correlation dimension $D_{2}$ is the slope of the line. For deterministic dynamics, with the increase of $m, D_{2}$ will tend to be saturated. This shows that there exists a fractal time series distribution characteristic and the attractor dimension $\mathrm{D}_{2}$ is equal to the linear slope.

2.2. Chaotic Diagnosis of Actuator Acceleration Signals. Based on formula (3), the time series of the actuator acceleration signals basically satisfies the independent requirements of dimensional phase space when the lag time $\tau=2$. And G-P method is applied to calculate the corresponding correlation dimension; thus the results are shown in Figure 3.

As shown in Figure 3, the correlation dimensions of the six actuators are 5, 8, 6, 5, 7, and 6, respectively; the results represent that there are 5, 8, 6, 5, 7, and 6 unknown impact factors on acceleration signals; thus the chaos system is 


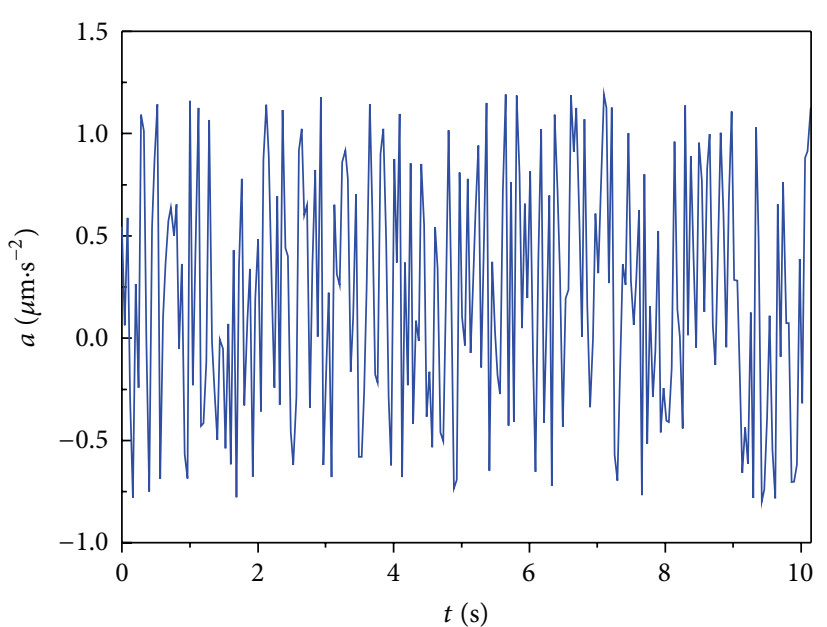

(a) Actuator 1

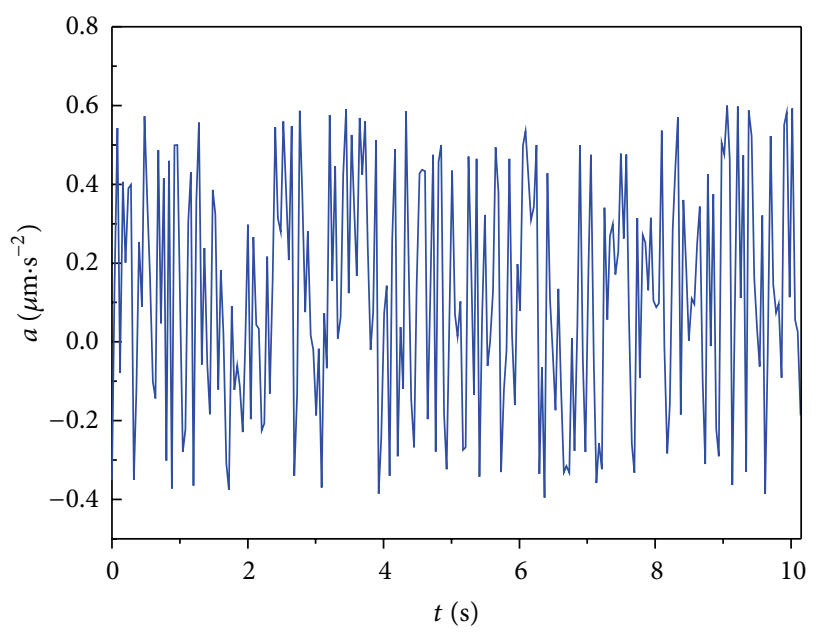

(c) Actuator 3

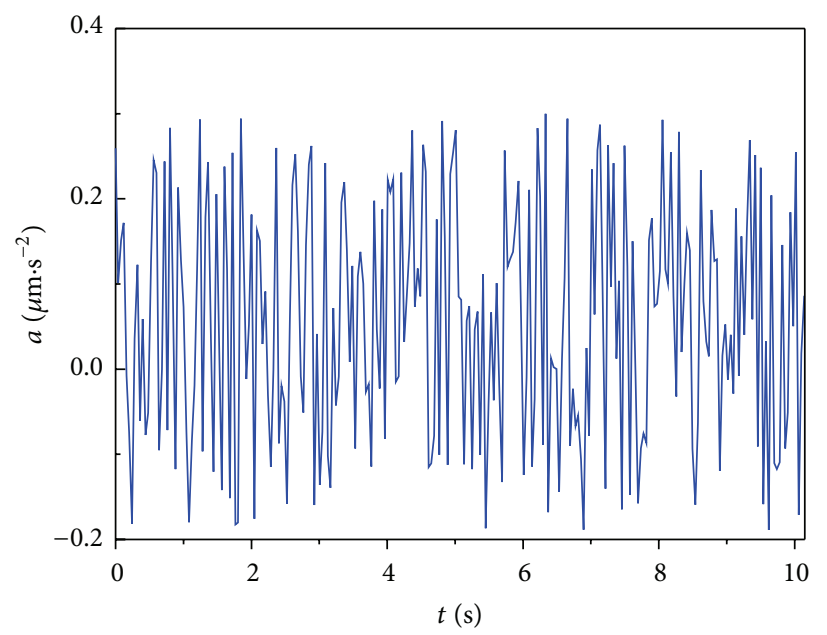

(e) Actuator 5

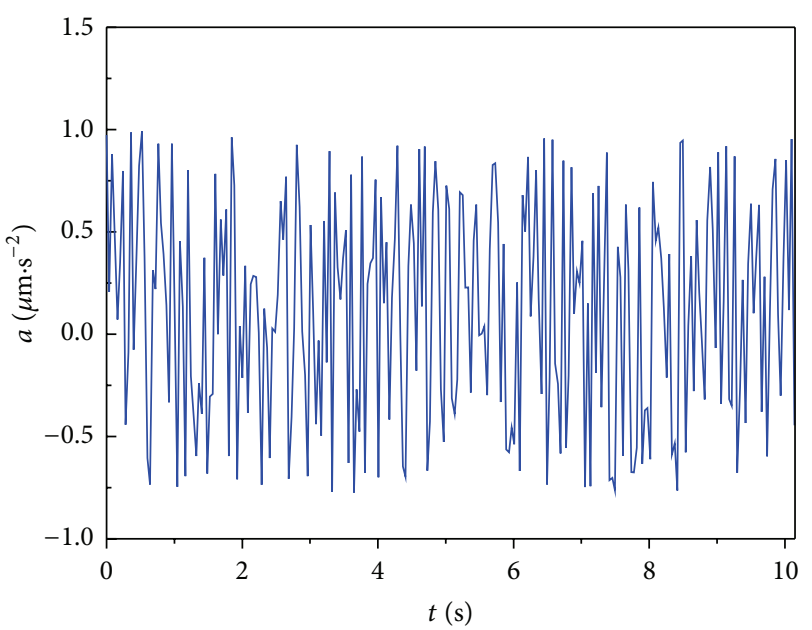

(b) Actuator 2

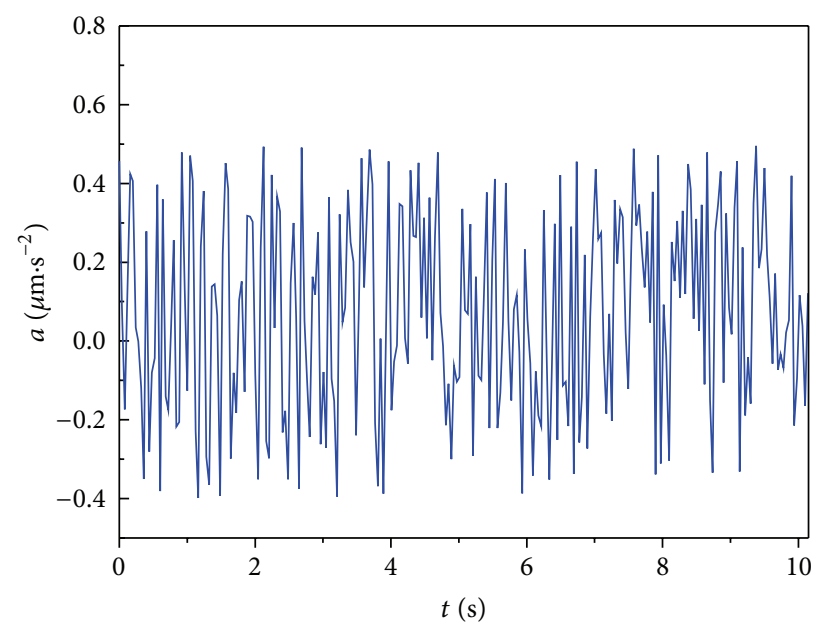

(d) Actuator 4

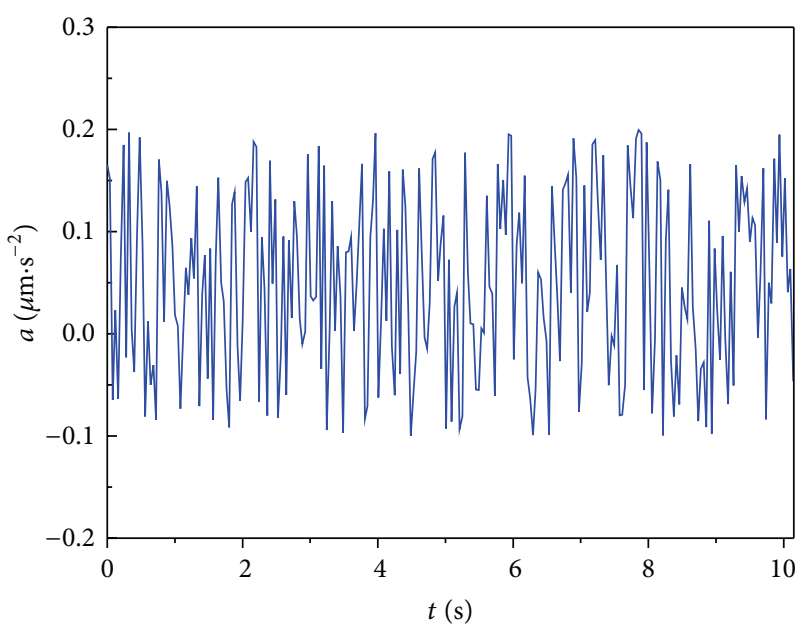

(f) Actuator 6

Figure 2: Acceleration signals of the actuators.

determined. With the increase of the embedding dimension of the time series of acceleration signals, $\ln (\varepsilon) \sim \ln \left[C_{m}(\varepsilon)\right]$ curve has linear correlation regional; therefore, the time series of acceleration signals has chaotic characteristics.
The slopes of curves are obtained after the least-squares fitting through the acceleration of time sequence correlation dimension figure. Figure 4 shows the relationship between the embedding dimension $m$ and correlation dimension $D_{2}$. 

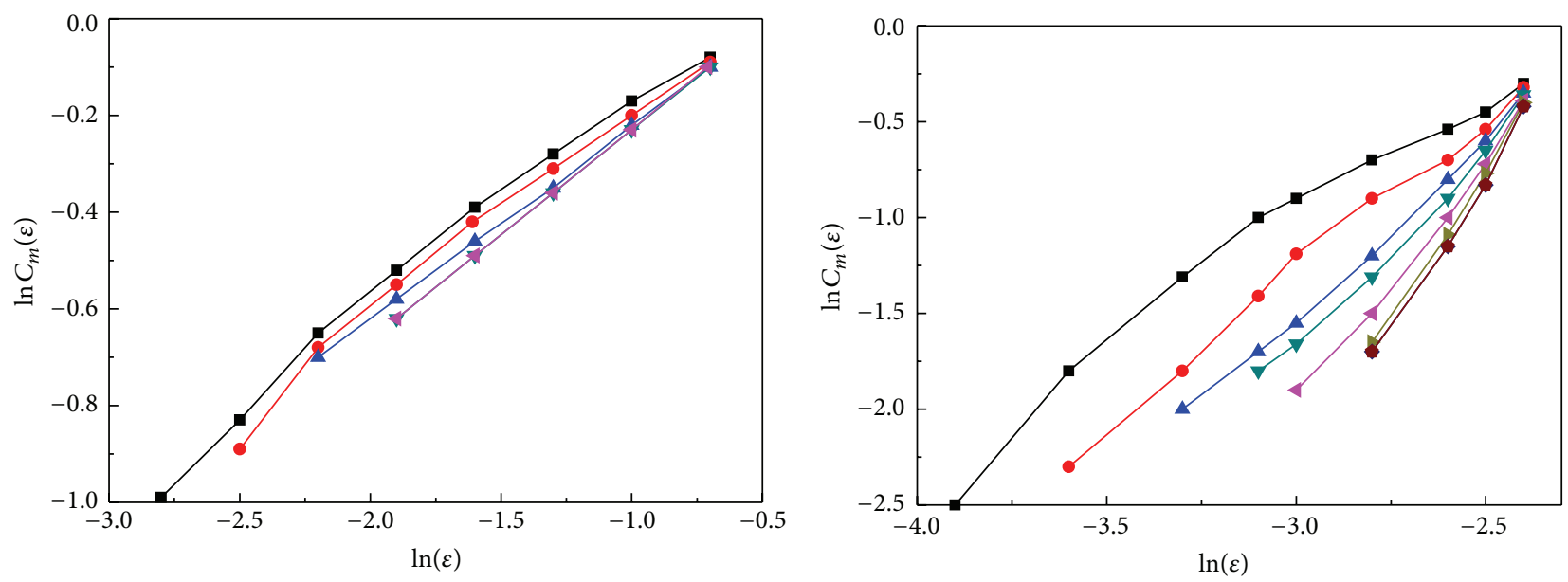

$\begin{array}{ll}\rightarrow m=1 & -m=3 \\ \rightarrow m=2 & \neg m=4\end{array}$

(a) Actuator 1

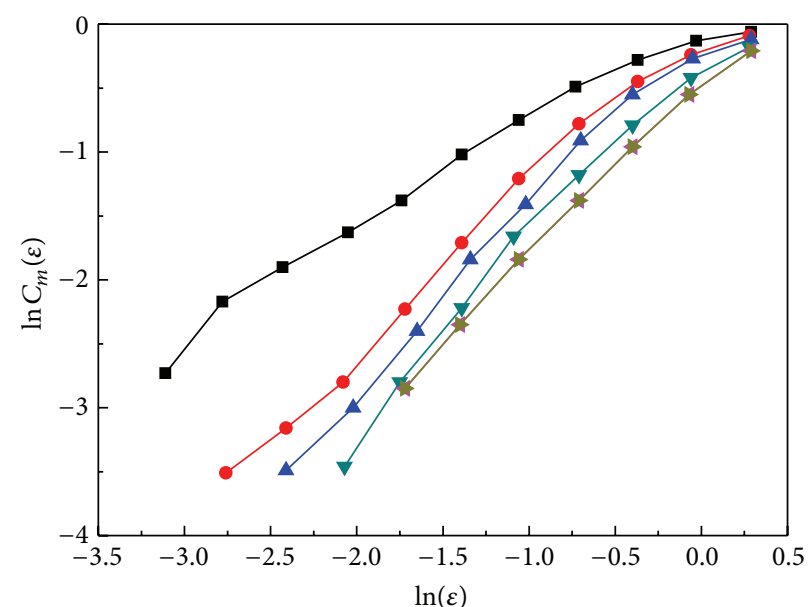

$\rightarrow-m=1$

$\longrightarrow m=2$

$\simeq m=3$

$\rightarrow m=4$

(c) Actuator 3

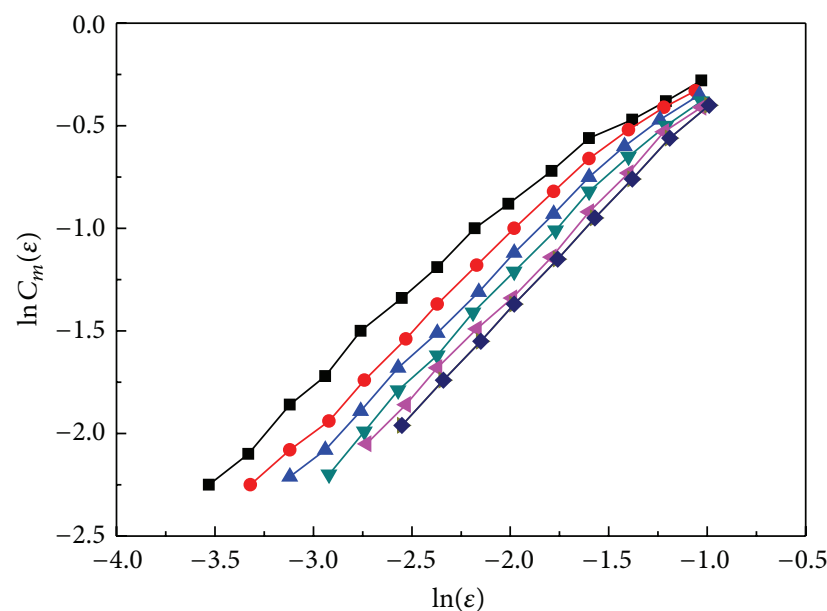

$\rightarrow m=1 \quad \neg m=3 \quad \longleftarrow m=5 \quad \neg m=7$

$\because m=2 \quad \neg-m=4 \quad \rightarrow m=6$

(e) Actuator 5

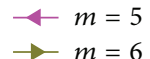

$\rightarrow m=6$

$$
1
$$

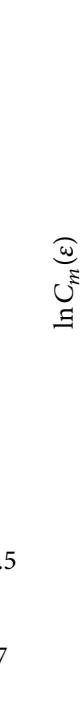

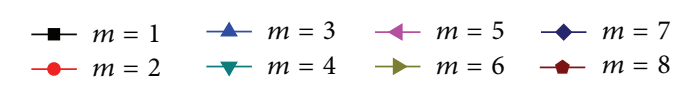

(b) Actuator 2

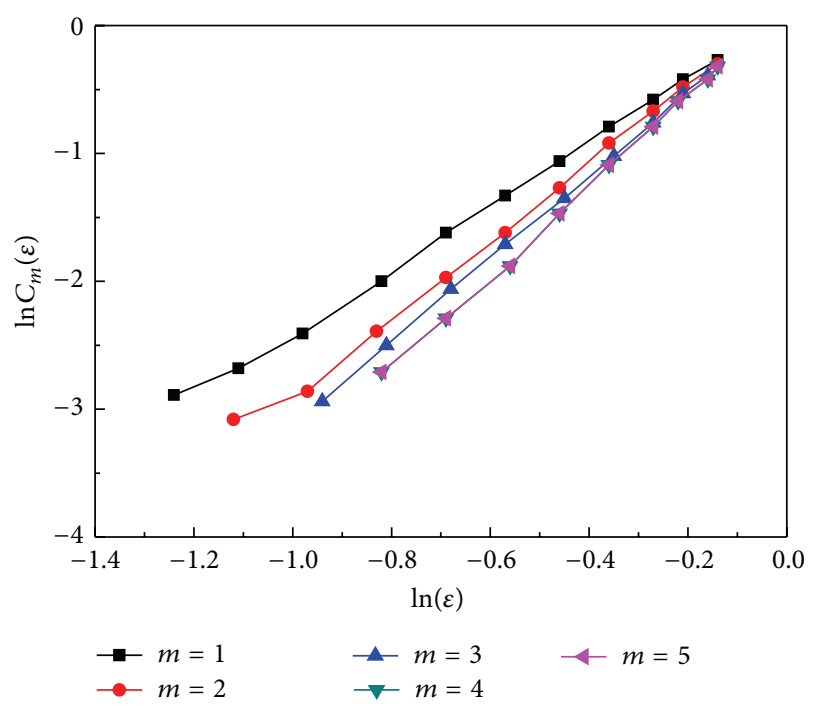

(d) Actuator 4

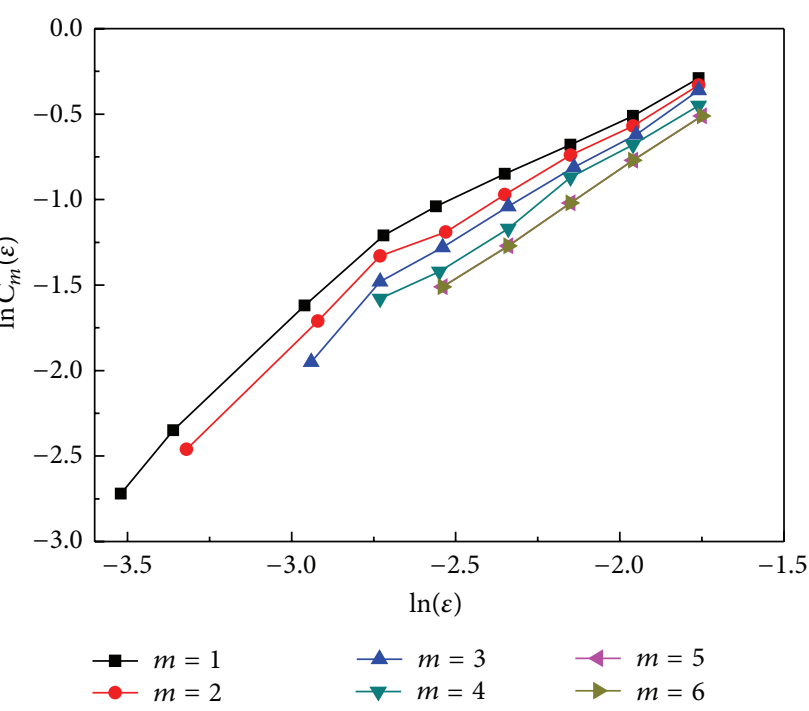

(f) Actuator 6

FIGURE 3: Correlation dimension on acceleration signals of the actuators. 


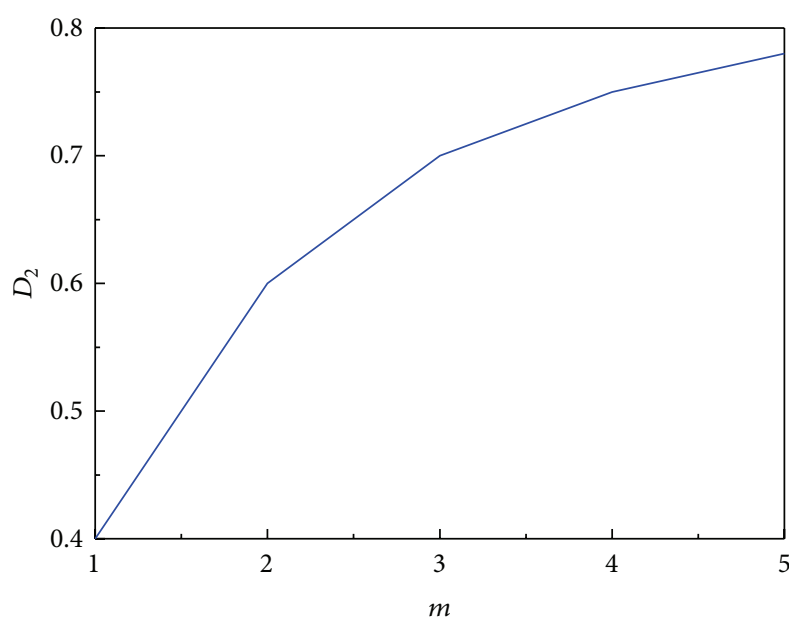

(a) Actuator 1

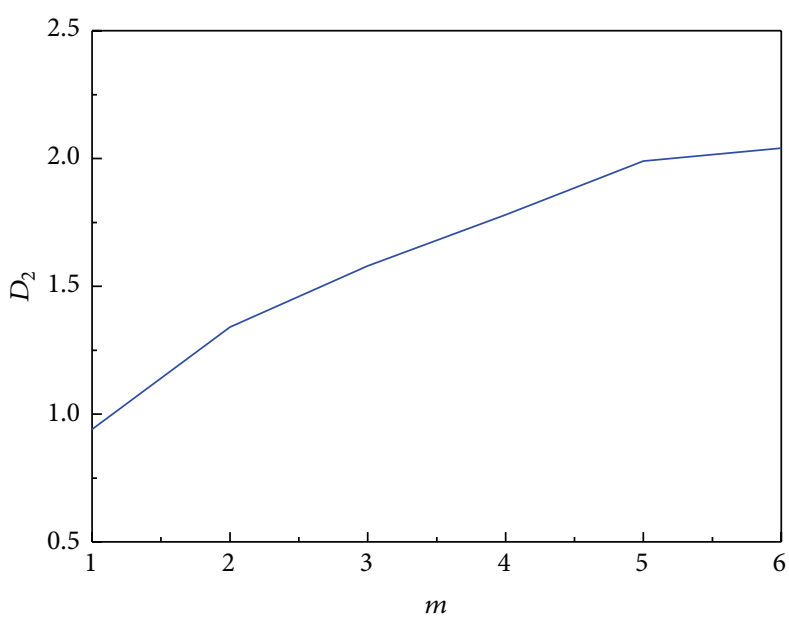

(c) Actuator 3

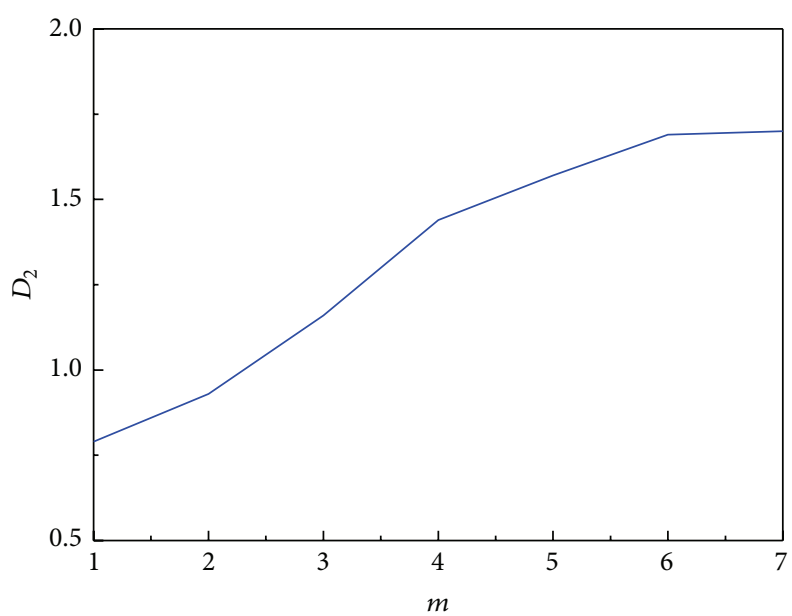

(e) Actuator 5

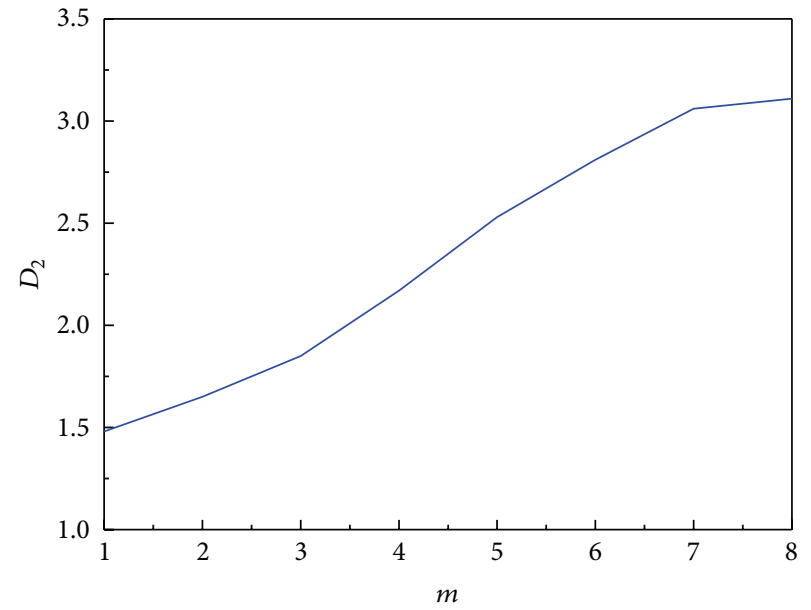

(b) Actuator 2

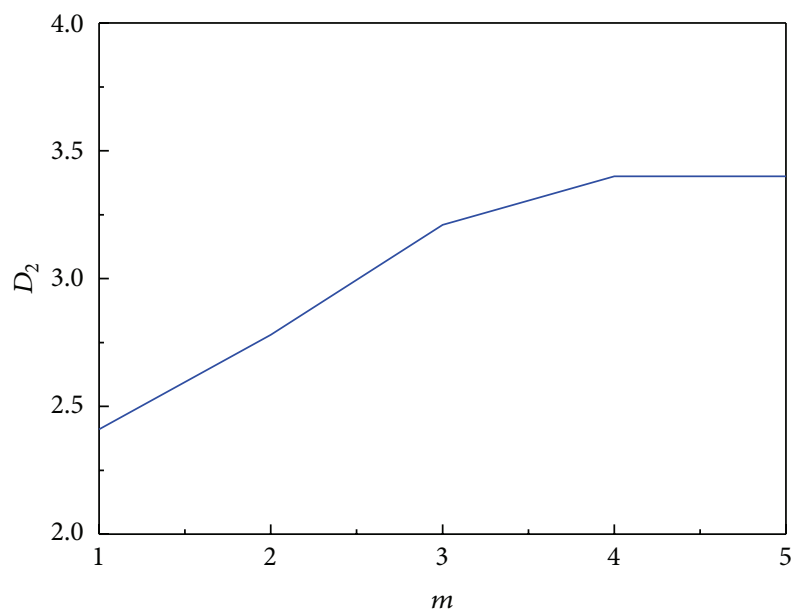

(d) Actuator 4

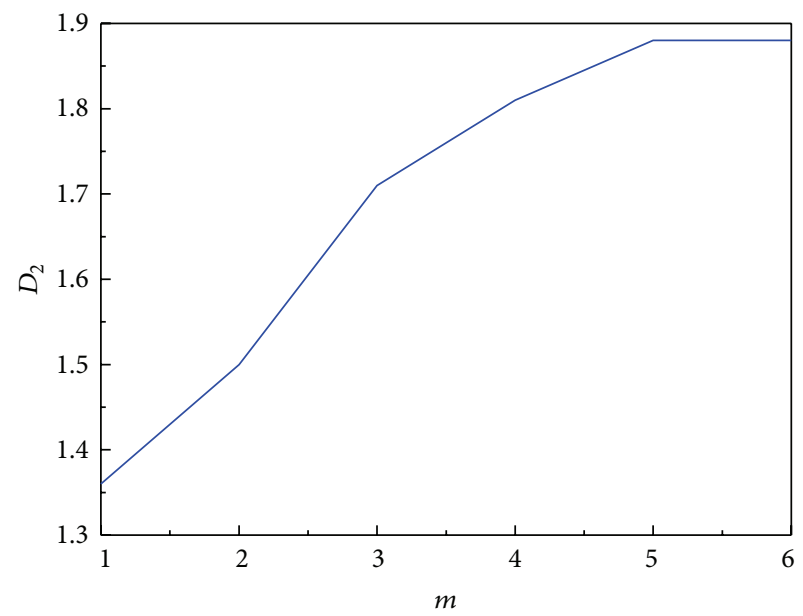

(f) Actuator 6

FIgURE 4: Change curve of the embedding dimension $m$ and saturated correlation dimension $D_{2}$.

According to Figures 3 and 4, some results can be concluded as follows: (1) When the acceleration time series of the embedding phase space dimension $m$ of actuator 1 are greater than or equal to 5 , the correlation dimension tends to be stable, and the corresponding saturated correlation dimension $D_{2}=0.78$. (2) When the acceleration time series of the embedding phase space dimension $m$ of actuator 2 are greater than or equal to 8 , the correlation dimension tends 
to be stable, and the corresponding saturated correlation dimension $D_{2}=3.11$. (3) When the acceleration time series of the embedding phase space dimension $m$ of actuator 3 are greater than or equal to 6 , the correlation dimension tends to be stable, and the corresponding saturated correlation dimension $D_{2}=2.04$. (4) When the acceleration time series of the embedding phase space dimension $m$ of actuator 4 are greater than or equal to 5 , and the correlation dimension tends to be stable, the corresponding saturated correlation dimension $D_{2}=3.4$. (5) When the acceleration time series of the embedding phase space dimension $m$ of actuator 5 are greater than or equal to 7 , the correlation dimension tends to be stable, and the corresponding saturated correlation dimension $D_{2}=1$.7. (6) When the acceleration time series of the embedding phase space dimension $m$ of actuator 6 are greater than or equal to 6 , the correlation dimension tends to be stable, and the corresponding saturated correlation dimension $D_{2}=1.88$.

2.3. Maximum Lyapunov Index of the Acceleration Time Series. The calculation of the saturated correlation dimension is a necessary condition to discriminate whether the acceleration series are chaotic or not. To determine whether the system is really chaotic, a more critical judgment of maximum Lyapunov index needs to be computed, which reflects the system adjacent track in the phase space of convergence and divergence of the long-term average. For maximum Lyapunov index time series, when $\lambda<0$, the system has a stable fixed point; when $\lambda=0$, it corresponds to the bifurcation point or system periodic solution of the system for the cycle system. When $\lambda>0$, it shows that the system is chaotic, and the greater the value of the index of the system, the more obvious the chaos characteristic and the higher the degree of chaos it represents.

The calculation process of maximum Lyapunov index is shown below $[28,29]$.

After reconstructing actuator acceleration signals in phase space, the initial shortest distance between each point is calculated and separated in a short time due to

$$
d_{i}(0)=\min \left\|X_{i}-X_{\hat{i}}\right\|, \quad|i-\hat{i}|>w,
$$

where $w$ represents the sequence of the sampling period, and it can be obtained by Fourier transform estimates

$$
w=\sum_{j=1}^{L} A_{j}\left(\sum_{j=1}^{L} f_{j} A_{j}\right)^{-1}
$$

where $f_{j}$ is evenly distributed between 0 and Nyquist frequency point; $A_{j}$ is amplitude corresponding to $f_{j}$.

For each point $X_{i}$ in the phase space, calculate the near point of the distance after $j$ discrete time step:

$$
\begin{aligned}
d_{i}(j)=\left\|X_{i+j}-X_{\hat{i}+j}\right\|, & \\
& j=1,2, \ldots, \min (n-i, n-\widehat{i}) .
\end{aligned}
$$

TABLE 1: Maximum Lyapunov index of the acceleration time series.

\begin{tabular}{lcc}
\hline Time series & Maximum Lyapunov index & Is there chaos? \\
\hline Actuator 1 & 0.0873 & Yes \\
Actuator 2 & 0.0694 & Yes \\
Actuator 3 & 0.0932 & Yes \\
Actuator 4 & 0.0739 & Yes \\
Actuator 5 & 0.0833 & Yes \\
Actuator 6 & 0.0497 & Yes \\
\hline
\end{tabular}

Assuming the $i$ th is the adjacent point approximation to maximum Lyapunov index rate index, thus

$$
d_{i}(j)=C_{i} e^{\lambda_{1}(j \Delta t)},
$$

where $C_{i}$ is the initial separation distance and it is constant.

After taking logarithm into both ends of (10), (11) can be obtained:

$$
\ln d_{i}(j)=\ln C_{i}+\lambda_{i}(j \Delta t), \quad i=1,2, \ldots, n .
$$

The equation represents a collection of approximate parallel lines; the slope was $\lambda_{1}$. After the least-squares fitting, the maximum Lyapunov index of $\lambda_{1}$ is expressed as

$$
\lambda_{1}=\frac{1}{\sum j^{2}} \sum[j * y(j)]
$$

where $y(i)$ can be expressed as

$$
y(i)=\frac{1}{p \Delta t} \sum_{i=1}^{p} \ln d_{i}(j),
$$

where $p$ is the number of nonzero $d_{i}(j)$.

Through calculating the maximum Lyapunov indexes of the acceleration time sequence of six actuators, the results are shown in Table 1.

From Table 1, the six acceleration time series are of obvious chaos characteristic, which means that there are lots of influencing factors to the acceleration variables.

2.4. Kolmogorov Entropies of Acceleration Time Series. Kolmogorov entropies [30-32] are proposed by Kolmogorov based on information entropy; the value of $K$ entropy can distinguish regular motion from chaotic system and random motion. When $K$ entropy is greater than 0 , it can be used to identify the chaotic characteristics of dynamic system and describe the chaos degree of dynamics. The bigger value of $K$ entropy means the larger loss of information rate; meanwhile, the bigger the chaos degree of the system is, the more complex the system is. Kolmogorov entropy is defined as the average loss rate of information, and it is difficult to calculate the value of Kolmogorov entropy in practical application since the dynamic equations of the system are impossible to be established correctly. However, the association of Kolmogorov entropy with Renyi entropy and the topological entropy can be expressed [32]:

$$
K_{2} \leq K_{1} \leq K_{0},
$$

where $K_{2}$ is Renyi entropy; $K_{1}$ is Kolmogorov entropy; $K_{0}$ is topological entropy. 
TABLE 2: Result of Kolmogorov entropy.

\begin{tabular}{lc}
\hline Time series & Kolmogorov entropy (nats/s) \\
\hline Actuator 1 & 0.081 \\
Actuator 2 & 0.216 \\
Actuator 3 & 0.107 \\
Actuator 4 & 0.088 \\
Actuator 5 & 0.109 \\
Actuator 6 & 0.188 \\
\hline
\end{tabular}

Therefore, Renyi entropy is usually used to calculate the approximate of Kolmogorov entropy [32]

$$
K_{2}(m, \varepsilon)=\frac{1}{\tau} \ln \frac{C_{m}(\varepsilon)}{C_{m+1}(\varepsilon)}
$$

where $C_{m}(\varepsilon)$ represents the integral function in the correlation dimension of (4).

The calculation results of Kolmogorov entropy are obtained in Table 2.

As shown in Table 2, it can be concluded that the six values of Kolmogorov entropy are small, among which the smaller one represents a lower degree of chaos while the bigger one represents a higher degree of chaos. By comparing $K$ entropy and correlation dimension, it can be found that the two are corresponding.

\section{Conclusion}

(1) The acceleration time series of the piezoelectric actuators are of chaotic character, and the time series is a chaotic sequence.

(2) When the lag time $\tau=2$, the acceleration time series of the piezoelectric actuators basically satisfies the independent requirements of the phase space of each dimension.

(3) The acceleration time series of the piezoelectric actuators result from the nonlinear chaotic dynamic system evolution, and the maximum or the minimum of the internal factors affecting acceleration time series of the piezoelectric actuators is 6 or 1 .

(4) The maximum Lyapunov indexes and Kolmogorov entropy of the acceleration time series of the piezoelectric actuators are solved and calculated, and the results show that the maximum Lyapunov indexes and Kolmogorov entropies are positive and the acceleration signals of the actuators are of chaos characteristics.

\section{Conflict of Interests}

The authors declare that there is no conflict of interests regarding the publication of this paper.

\section{Acknowledgments}

The authors would like to acknowledge Project 9140A2011QT4801 supported by Weapons and Equipment Pre-Research Fund and Project K1205117-11 supported by Key Project of Changsha Science and Technology Project.

\section{References}

[1] H. S. Choi and J. Y. K. Lou, "Nonlinear behaviour of an articulated offshore loading platform," Applied Ocean Research, vol. 13, no. 2, pp. 63-74, 1991.

[2] Y. Ting, C.-C. Li, and T. V. Nguyen, "Composite controller design for a 6DOF Stewart nanoscale platform," Precision Engineering, vol. 37, no. 3, pp. 671-683, 2013.

[3] L. Fraguela, L. Fridman, and V. V. Alexandrov, "Output integral sliding mode control to stabilize position of a Stewart platform," Journal of the Franklin Institute, vol. 349, no. 4, pp. 1526-1542, 2012.

[4] J. E, C. Qian, T. Liu, and G.-L. Liu, "Research on the vibration characteristics of the new type of passive super static vibratory platform based on the multiobjective parameter optimization," Advances in Mechanical Engineering, vol. 6, Article ID 569289, 2014.

[5] C.-W. Chang-Jian and S.-M. Chang, "Bifurcation and chaos analysis of spur gear pair with and without nonlinear suspension," Nonlinear Analysis: Real World Applications, vol. 12, no. 2, pp. 979-989, 2011.

[6] H. Q. Li, X. F. Liao, S. Ullah, and L. Xiao, "Analytical proof on the existence of chaos in a generalized Duffing-type oscillator with fractional-order deflection," Nonlinear Analysis. Real World Applications, vol. 13, no. 6, pp. 2724-2733, 2012.

[7] L.-S. Hu, X.-J. Wang, G.-S. Yu et al., "Chaotic analysis of pressure fluctuation signal in the gas-liquid-solid slurry column," Nonlinear Analysis: Real World Applications, vol. 10, no. 1, pp. 410-415, 2009.

[8] F. Gao, X.-J. Lee, F.-X. Fei, H.-Q. Tong, Y.-F. Deng, and H.-L. Zhao, "Identification time-delayed fractional order chaos with functional extrema model via differential evolution," Expert Systems with Applications, vol. 41, no. 4, pp. 1601-1608, 2014.

[9] A. Farshidianfar and A. Saghafi, "Identification and control of chaos in nonlinear gear dynamic systems using Melnikov analysis," Physics Letters A, vol. 378, no. 46, pp. 3457-3463, 2014.

[10] E. Gaume, B. Sivakumar, M. Kolasinski, and L. Hazoumé, "Identification of chaos in rainfall temporal disaggregation: application of the correlation dimension method to 5-minute point rainfall series measured with a tipping bucket and an optical raingage," Journal of Hydrology, vol. 328, no. 1-2, pp. 5664, 2006.

[11] E. M. Miandoab, A. Yousefi-Koma, H. N. Pishkenari, and F. Tajaddodianfar, "Study of nonlinear dynamics and chaos in MEMS/NEMS resonators," Communications in Nonlinear Science and Numerical Simulation, vol. 22, no. 1-3, pp. 611-622, 2015.

[12] E. M. Miandoab, H. N. Pishkenari, A. Yousefi-Koma, and F. Tajaddodianfar, "Chaos prediction in MEMS-NEMS resonators," International Journal of Engineering Science, vol. 82, pp. 74-83, 2014.

[13] R. Khatibi, B. Sivakumar, M. A. Ghorbani, O. Kisi, K. Koçak, and D. F. Zadeh, "Investigating chaos in river stage and discharge time series," Journal of Hydrology, vol. 414-415, pp. 108-117, 2012. 
[14] C.-M. Chang and Z.-M. Ge, "Complete identification of chaos of nonlinear nonholonomic systems," Nonlinear Dynamics, vol. 60, no. 4, pp. 551-559, 2010.

[15] L. G. Yuan, Q. G. Yang, and C. B. Zeng, "Chaos detection and parameter identification in fractional-order chaotic systems with delay," Nonlinear Dynamics, vol. 73, no. 1-2, pp. 439-448, 2013.

[16] C. Luo and X. Y. Wang, "Chaos in the fractional-order complex Lorenz system and its synchronization," Nonlinear Dynamics, vol. 71, no. 1-2, pp. 241-257, 2013.

[17] G. M. Mahmoud, T. Bountis, G. M. AbdEl-Latif, and E. E. Mahmoud, "Chaos synchronization of two different chaotic complex Chen and Lü systems," Nonlinear Dynamics, vol. 55, no. 1-2, pp. 43-53, 2009.

[18] G.-C. Wu and D. Baleanu, "Discrete fractional logistic map and its chaos," Nonlinear Dynamics, vol. 75, no. 1-2, pp. 283-287, 2014.

[19] D. Ruelle and F. Takens, "On the nature of turbulence," Communications in Mathematical Physics, vol. 20, no. 3, pp. 167-192, 1971.

[20] H. Li, X. Liao, J. Huang, G. Chen, Z. Dong, and T. Huang, "Diverting homoclinic chaos in a class of piecewise smooth oscillators to stable periodic orbits using small parametrical perturbations," Neurocomputing, vol. 149, pp. 1587-1595, 2015.

[21] H. Li, X. Liao, and R. Liao, "A unified approach to chaos suppressing and inducing in a periodically forced family of nonlinear oscillators," IEEE Transactions on Circuits and Systems. I. Regular Papers, vol. 59, no. 4, pp. 784-795, 2012.

[22] H. Li, X. Liao, X. Lei, T. Huang, and W. Zhu, "Secondorder consensus seeking in multi-agent systems with nonlinear dynamics over random switching directed networks," IEEE Transactions on Circuits and Systems -I: Regular Papers, vol. 60, no. 6, pp. 1595-1607, 2013.

[23] H. Li, X. Liao, T. Huang, Y. Wang, Q. Han, and T. Dong, "Algebraic criteria for second-order global consensus in multiagent networks with intrinsic nonlinear dynamics and directed topologies," Information Sciences, vol. 259, no. 1, pp. 25-35, 2014.

[24] C. H. Wang, X. W. Luo, and Z. Wan, "Generation and circuit implementation of multi-block multidirectional grid multiscroll chaotic attractors," Optik, vol. 125, no. 22, pp. 6716-6721, 2014.

[25] D. Kim and P. H. Chang, "A new butterfly-shaped chaotic attractor," Results in Physics, vol. 3, pp. 14-19, 2013.

[26] L. Pan, W. N. Zhou, and J. A. Fang, "On dynamics analysis of a novel three-scroll chaotic attractor," Journal of the Franklin Institute, vol. 347, no. 2, pp. 508-522, 2010.

[27] F. Takens, Dynamical System and Turbulence, Lecture Notes in Mathematics, Springer, New York, NY, USA, 1981.

[28] P. Balenzuela and C. O. Dorso, "Maximum Lyapunov exponent of highly excited finite systems," Physica A: Statistical Mechanics and Its Applications, vol. 283, no. 1-2, pp. 267-272, 2000.

[29] W. Caesarendra, B. Kosasih, A. K. Tieu, and C. A. S. Moodie, "Application of the largest Lyapunov exponent algorithm for feature extraction in low speed slew bearing condition monitoring," Mechanical Systems and Signal Processing, vol. 50-51, pp. 116-138, 2015.

[30] X. L. Jia, C. D. Zhao, and X. B. Yang, "Global attractor and Kolmogorov entropy of three component reversible GrayScott model on infinite lattices," Applied Mathematics and Computation, vol. 218, no. 19, pp. 9781-9789, 2012.
[31] L. I. Aftanas, N. V. Lotova, V. I. Koshkarov, V. L. Pokrovskaja, S. A. Popov, and V. P. Makhnev, "Non-linear analysis of emotion EEG: calculation of Kolmogorov entropy and the principal Lyapunov exponent," Neuroscience Letters, vol. 226, no. 1, pp.1316, 1997.

[32] J. Q. E, Y. N. Wang, C. Mei, and J. K. Gong, "Chaotic behavior of crude copper composition time series in the process of matte converting and its predicable time scale," Nonlinear Analysis: Real World Applications, vol. 7, no. 4, pp. 651-661, 2006. 


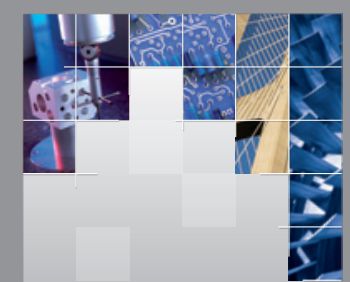

\section{Enfincering}
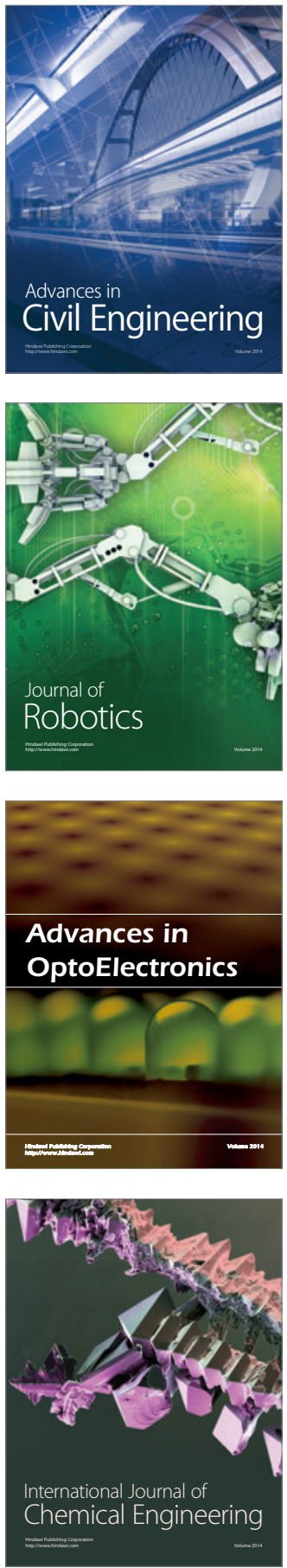

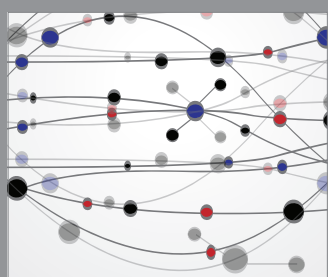

The Scientific World Journal

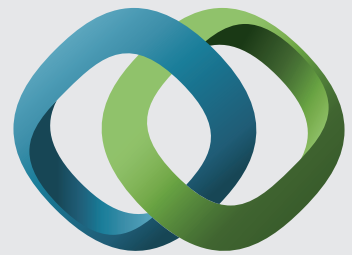

\section{Hindawi}

Submit your manuscripts at

http://www.hindawi.com
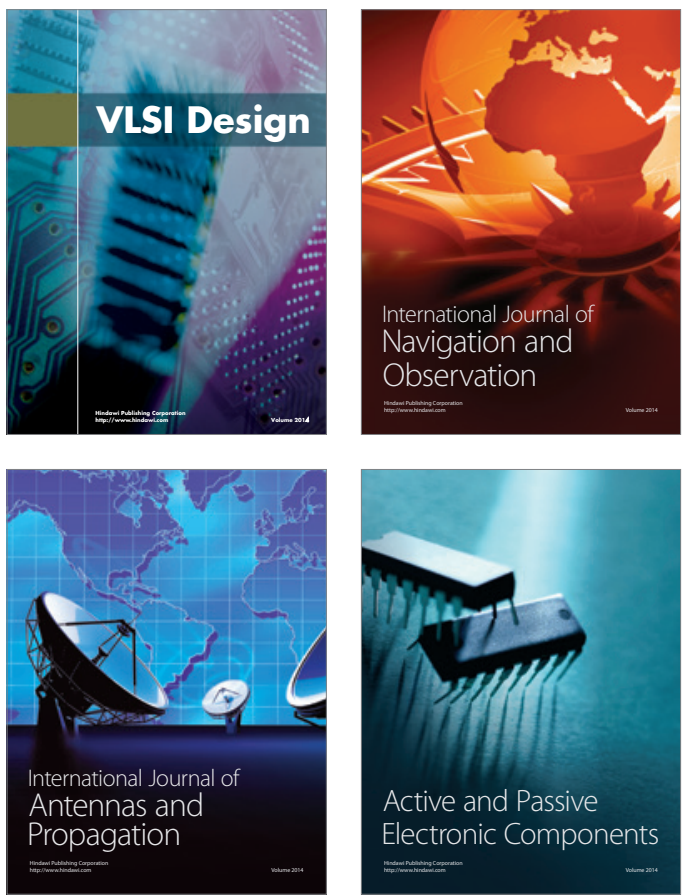
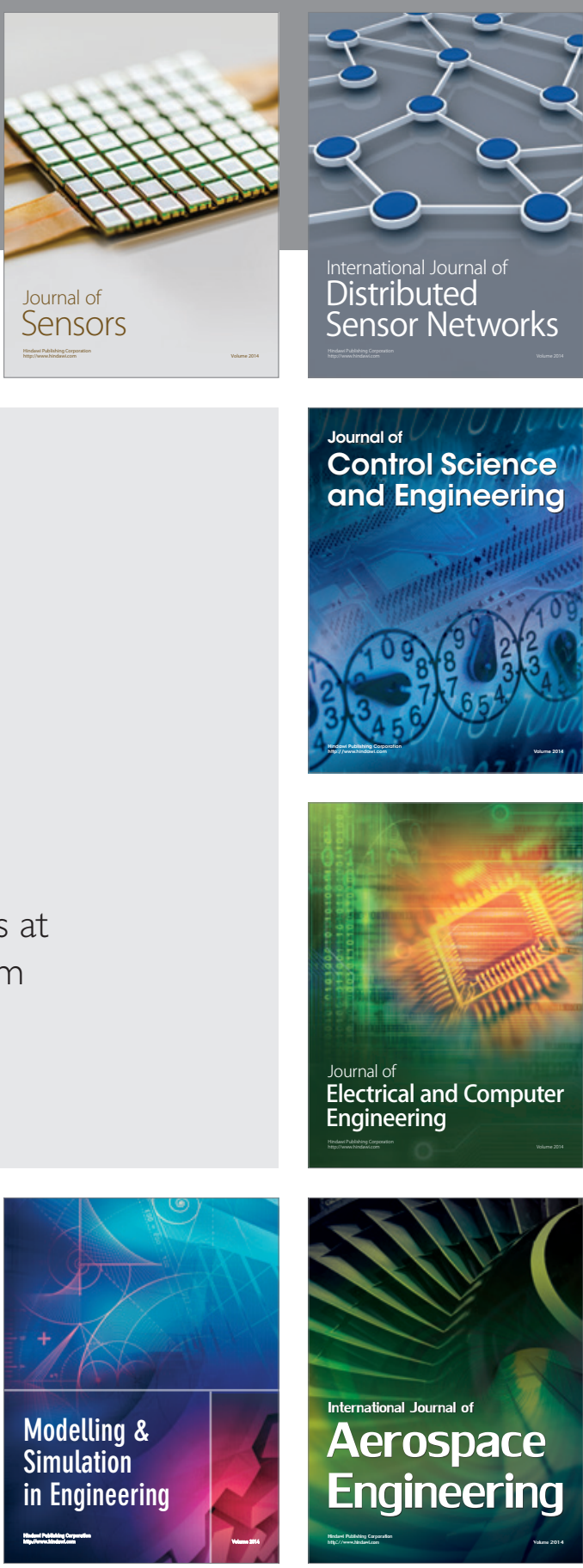

International Journal of

Distributed

Sensor Networks

Journal of

Control Science

and Engineering
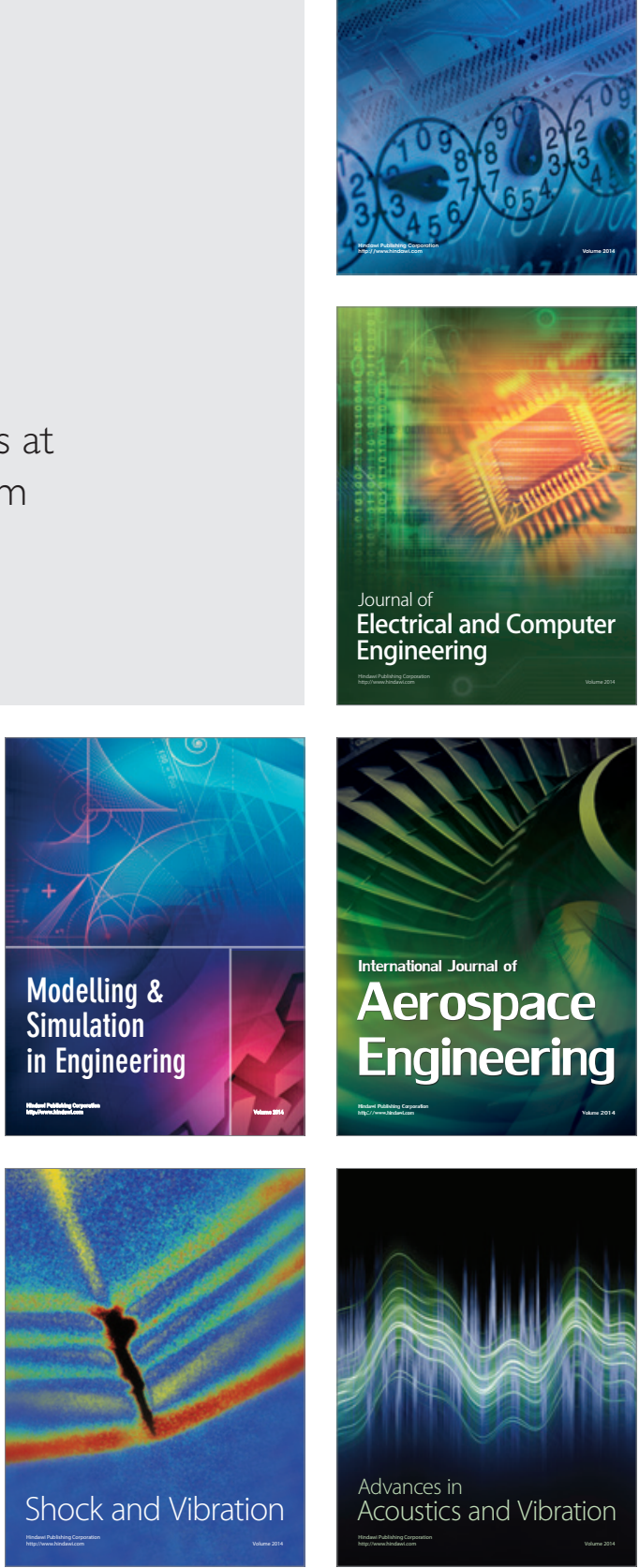